\title{
Magnetic proximity effect at the three-dimensional topological insulator/magnetic insulator interface
}

\author{
S. V. Eremeev, ${ }^{1,2}$ V. N. Men'shov, ${ }^{2,3}$ V. V. Tugushev, ${ }^{2,3,4}$ P. M. Echenique, ${ }^{5,6}$ and E. V. Chulkov ${ }^{2,5,6}$ \\ ${ }^{1}$ Institute of Strength Physics and Materials Science, 634021 Tomsk, Russia \\ ${ }^{2}$ Tomsk State University, 634050 Tomsk, Russia \\ ${ }^{3}$ NRC Kurchatov Institute, Kurchatov Sqr. 1, 123182 Moscow, Russia \\ ${ }^{4}$ Prokhorov General Physics Institute, Vavilov Str. 38, 119991 Moscow, Russia \\ ${ }^{5}$ Donostia International Physics Center (DIPC), 20018 San Sebastián/Donostia, Basque Country, Spain \\ ${ }^{6}$ Departamento de Física de Materiales UPVIEHU, Centro de Física de Materiales CFM-MPC and Centro Mixto CSIC-UPVIEHU, \\ 20080 San Sebastián/Donostia, Basque Country, Spain
}

(Received 20 March 2013; revised manuscript received 5 October 2013; published 31 October 2013)

\begin{abstract}
The magnetic proximity effect is a fundamental feature of heterostructures composed of layers of topological insulators and magnetic materials since it underlies many potential applications in devices with novel quantum functionality. Within density functional theory we study magnetic proximity effect at the three-dimensional topological insulator/magnetic insulator (TI/MI) interface in the $\mathrm{Bi}_{2} \mathrm{Se}_{3} / \mathrm{MnSe}(111)$ system as an example. We demonstrate that a gapped ordinary bound state caused by the interface potential arises in the immediate region of the interface. The gapped topological Dirac state also arises in the system owing to relocation to deeper atomic layers of topological insulator. The gap in the Dirac cone originates from an overlapping of the topological and ordinary interfacial states. This result being also corroborated by the analytic model, is a key aspect of the magnetic proximity effect mechanism in the TI/MI structures.
\end{abstract}

DOI: 10.1103/PhysRevB.88.144430

PACS number(s): 73.20.-r, 75.70.Tj, 85.75.-d

\section{INTRODUCTION}

It is well known that, in the three-dimensional (3D) topological insulator (TI) based nanostructures unusual ("topological") bound states, also called Dirac helical states, arise at the boundary between the topologically nontrivial and trivial components of structure. These states have many exciting physical properties supported by the time-reversal symmetry of TI, such as linear gapless dispersion and spinmomentum locking. ${ }^{1}$ To efficiently realize the potential of 3D TIs in spin electronic and magnetic storage applications, it is advisable to integrate the nonmagnetic 3D TI layers into hybrid heterostructures containing the layers of ferromagnetic (FM) or antiferromagnetic (AFM) materials. ${ }^{2-4}$ Due to the time-reversal symmetry breaking in such structures, the effect of magnetism on the topological states provides opportunities for various nanoelectronic device applications using magnetic and magnetotransport features of the helical quasiparticles, in particular, the effect of proximity-induced ferromagnetism ${ }^{5}$ and quantum anomalous Hall effect. ${ }^{6}$

One has to tune the 3D TI/FM (AFM) heterostructures in such a way that the spectrum of topological states could be easily accessible to an exchange field influence of the FM (AFM) layer without significant spin dependent scattering of these states on magnetic ions. At the same time, the spin dependent transport of carriers in 3D TI should be controllable. These demands are not easily feasible because of a number of obstacles. On the one hand, a serious problem is to find the FM or AFM material which forms a high-quality interface with the TI material and at the same time provides a strong magnetic interaction with it. On the other hand, the physics of the exchange coupling at the TI/FM (AFM) boundary is not yet well understood. In principle, there exist different possibilities to provide an exchange field influence from a magnetic material on the topological states in a 3D TI.
One way is to use the effect of the surface magnetic order in 3D TIs with chemisorbed magnetic impurities. While the local magnetic moments of impurities are arranged inside a thin layer with the thickness of the order of their diffusion length into the TI and form a magnetically ordered overlayer, the region of spin polarization of carriers near the 3D TI surface may be significantly larger due to magnetic proximity effect. This type of order can be realized by deposition of magnetic ions of $3 d$ metals ( $\mathrm{Mn}, \mathrm{Fe}, \mathrm{Cr}$, and $\mathrm{Co}$ ) on $\mathrm{Bi}_{2} \mathrm{Te}_{3}$, $\mathrm{Bi}_{2} \mathrm{Se}_{3}$, or $\mathrm{Sb}_{2} \mathrm{Te}_{3} .{ }^{7-10}$ When the surface concentration of ions is relatively high an indirect exchange coupling among their local magnetic moments mediated by the surface states of the 3D TI can arise. ${ }^{11,12}$ As a result the system becomes unstable with respect to FM order with a spontaneous magnetization along the normal axis, which is accompanied by opening a gap in a spectrum of the Dirac surface states. ${ }^{12-16}$ However, if the energy of exchange coupling is smaller than the inverse lifetime of the TI surface state due to impurity disorder scattering, both the FM ordering and the energy gap should be suppressed.

Another way to induce magnetic order on the surface of 3D TIs is coating with an external FM or AFM overlayer. This has been done in Ref. 17 where FM order was induced in $\mathrm{Bi}_{2-x} \mathrm{Mn}_{x} \mathrm{Te}_{3}(x=0.09)$ by a magnetic proximity effect through the deposited $\mathrm{Fe}$ overlayer in a temperature range well above the intrinsic Curie temperature of the bulk $\mathrm{Bi}_{2-x} \mathrm{Mn}_{x} \mathrm{Te}_{3}$. Unfortunately, in the case of metallic FM (AFM) materials the TI surface states near the TI/FM (AFM) interface should be significantly altered due to their hybridization with the bulk states of FM (AFM) metal. While density-functional theory (DFT) calculations are absent for this type of structure, one can suppose that the spectrum of the TI surface states should even lose helical features due to the alternation, since the density of states of the metallic FM 
(AFM) overlayer is much larger than the density of surface states of the 3D TI.

The film of the traditional FM (AFM) insulator (below called magnetic insulator, MI) adjacent to TI is a most promising candidate to manipulate the helical states of the 3D TI by means of magnetic proximity effect. ${ }^{18}$ Such a way may diminish the surface scattering via continuum states of the magnetic film, contrary to the metallic film case. Recently, several MIs (EuO, EuS, EuSe, MnSe, MnTe, and $\mathrm{RbMnCl}_{3}$ ) with compatible magnetic structure and relatively good lattice matching with TIs $\left(\mathrm{Bi}_{2} \mathrm{Te}_{3}, \mathrm{Bi}_{2} \mathrm{Se}_{3}\right.$, or $\left.\mathrm{Sb}_{2} \mathrm{Te}_{3}\right)$ have been identified, and the best candidate material was found to be the large gap AFM semiconductor MnSe. ${ }^{19}$ Note, however, that only for the system containing the $\mathrm{Bi}_{2} \mathrm{Se}_{3}$ and $\mathrm{EuS}$ layers the magnetic proximity effect has been experimentally demonstrated. ${ }^{5}$

In the present paper, we study the physics of magnetic proximity effect at the TI/MI interface by using first-principles DFT calculations and an analytical $\mathbf{k} \cdot \mathbf{p}$ model. As a model system we have chosen the $\mathrm{Bi}_{2} \mathrm{Se}_{3} / \mathrm{MnSe}(111)$ interface. We show that two types of interfacial bound states (referred to as the topological state and the interfacial ordinary state, respectively) appear at the TI side of the interface. These states have different physical origins, spatial distributions, and energy spectra. Namely, the topological state stems from a breaking of the $\mathbb{Z}_{2}$ invariant of the TI at the boundary with the MI. This state is located relatively far from the interface plane; its spectrum is gapped and lies inside the bulk energy gap of the TI. In contrast, the interfacial ordinary state results from the crystal symmetry breaking at the TI/MI interface. This state is located nearby the interface and is strongly spin polarized; its spectrum is gapped and lies far below the bulk energy gap of the TI due to the band bending at the TI side of the interface.

\section{METHODS}

For structural optimization and electronic band calculations we use the Vienna $A b$ Initio Simulation Package ${ }^{20,21}$ with generalized gradient approximation $(\mathrm{GGA})^{22}$ to the exchange correlation potential. The interaction between the ion cores and valence electrons was described by the projector augmentedwave method. ${ }^{23,24}$ The Hamiltonian contains scalar relativistic corrections, and the spin-orbit interaction (SOI) is taken into account by the second variation method. ${ }^{25}$ To correctly describe the highly correlated Mn- $d$ electrons we include the correlation effects within the GGA $+U$ method as developed in Ref. 26.

To simulate the $\mathrm{Bi}_{2} \mathrm{Se}_{3} / \mathrm{MnSe}(111)$ heterostructure, the in-plane lattice constant of the MnSe is fixed to that of $\mathrm{Bi}_{2} \mathrm{Se}_{3}$. The most stable structure of bulk $\mathrm{MnSe}$ is a cubic $\mathrm{NaCl}-$ type lattice, with antiferromagnetic ordering along the [111] direction. ${ }^{27}$ The optimization of MnSe with the fixed parameter in the (111) plane leads to $10 \%$ contraction of the $\mathrm{Mn}-\mathrm{Se}$ interlayer distance in the [111] direction. The typical values of correlation parameters $U=5.0 \mathrm{eV}$ and $J=1.0 \mathrm{eV}$ (Refs. 28 and 29) are appropriate for cubic MnSe, while for distorted $\mathrm{MnSe}$ they give Mn $d$ states within the gap. For this reason $U=6.0 \mathrm{eV}$, repelling $\mathrm{Mn} d$ bands into the conduction band used for the $\mathrm{Bi}_{2} \mathrm{Se}_{3} / \mathrm{MnSe}(111)$ heterostructure calculations.
It is important that the orthorhombic distortion of MnSe keeps the Mn magnetic moment equal to $\pm 4.58 \mu_{\mathrm{B}}$ (it is $\pm 4.57 \mu_{\mathrm{B}}$ in cubic structure with experimental lattice parameters) as well as antiferromagnetic ordering along the [111] direction. It means that in the direction perpendicular to the interface the orthorhombically distorted MnSe will provide the same magnetic exchange coupling as the cubic MnSe.

\section{RESULTS AND DISCUSSION}

The $\mathrm{Bi}_{2} \mathrm{Se}_{3} / \mathrm{MnSe}(111)$ interface was studied by constructing a superlattice composed of $\mathrm{Bi}_{2} \mathrm{Se}_{3}$ and $\mathrm{MnSe}$ slabs. Two types of interfaces with a Mn interfacial atomic layer are geometrically possible: fcc-type interface, where an interfacial $\mathrm{Mn}$ atom is situated in the fcc-hollow position on top of the $\mathrm{Bi}_{2} \mathrm{Se}_{3}$ slab [Fig. 1(a)] and hcp-type interface, where an interfacial $\mathrm{Mn}$ atom is situated in the hcp-hollow position (not shown). The constructed supercells contain two interfaces. To avoid the interface-interface interaction the slabs of seven quintuple layers (QLs) of $\mathrm{Bi}_{2} \mathrm{Se}_{3}$ and of 31 (33) atomic layers of MnSe were used for the fcc-type (hcp-type) interface. The interfacial space (separation distance between the $\mathrm{Bi}_{2} \mathrm{Se}_{3}$ and MnSe slabs) as well as the atomic positions within the first (closest to the interface) $\mathrm{QL}$ of $\mathrm{Bi}_{2} \mathrm{Se}_{3}$ and five near-interface

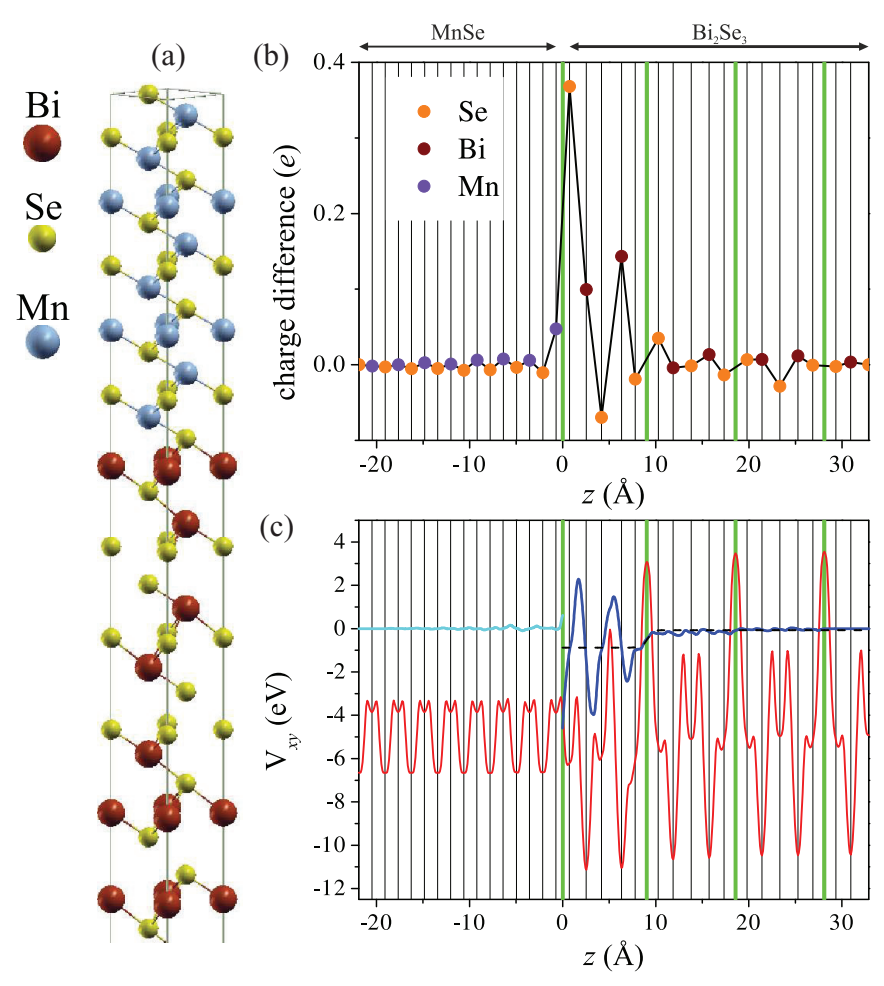

FIG. 1. (Color online) (a) Crystal structure of the $\mathrm{Bi}_{2} \mathrm{Se}_{3} / \mathrm{MnSe}$ (111) fcc-type interface (half of the supercell is shown); (b) atomic charge difference with respect to the electronic charge of bulklike atoms; (c) total electrostatic potential averaged over $x y$ planes $V_{x y}$ (red line) and change of the potential with respect to that in the central parts of the $\operatorname{MnSe}\left(\Delta V_{\mathrm{MI}}\right.$, light blue line) and $\mathrm{Bi}_{2} \mathrm{Se}_{3}\left(\Delta V_{\mathrm{TI}}\right.$, dark blue line $)$ slabs. The dashed line shows the Boltzmann fit for $\Delta V_{\mathrm{TI}}$. Vertical black lines mark the position of atomic layers; vertical green lines denote the borders of the $\mathrm{Bi}_{2} \mathrm{Se}_{3}$ QLs; $z=0$ corresponds to the interfacial plane. 


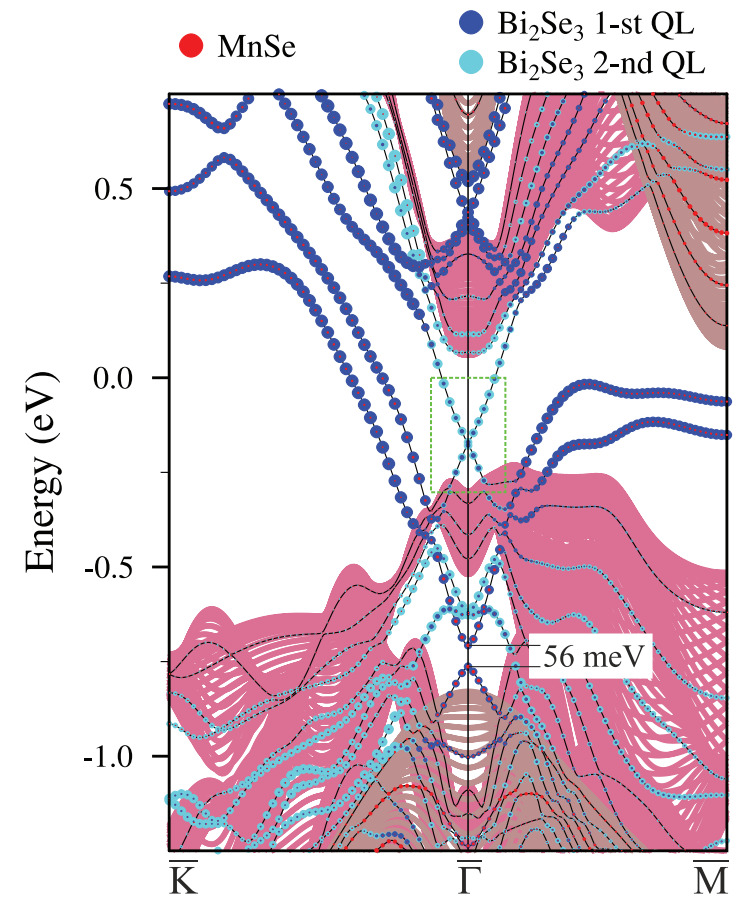

FIG. 2. (Color online) Electronic structure of the $\mathrm{Bi}_{2} \mathrm{Se}_{3} / \mathrm{MnSe}$ heterostructure. The size of the red circles corresponds to the weight of the states in the five atomic layers of MnSe adjacent to the interface; dark and light blue circles denote the weight of the states in the first and second QLs of $\mathrm{Bi}_{2} \mathrm{Se}_{3}$ near the interface. The projected bulk bands are shown in pale violet-red and rosy-brown for $\mathrm{Bi}_{2} \mathrm{Se}_{3}$ and MnSe, respectively.

atomic layers of MnSe were optimized while interatomic distances within the middle part of both slabs were fixed. The total energy optimization shows that the fcc-type interface gains an energy of $115 \mathrm{meV}$ with respect to the hcp-type heterostructure. For this reason in the following we will focus on the fcc-type $\mathrm{Bi}_{2} \mathrm{Se}_{3} / \mathrm{MnSe}(111)$ interface.

As far as the charge transfer and charge redistribution being the common feature for any interface we have estimated this effect by implementing the Bader charge analysis. ${ }^{30}$ In Fig. 1(b) the atomic charge difference with respect to the total electronic charge of central (bulklike) atoms of the $\mathrm{Bi}_{2} \mathrm{Se}_{3}$ and MnSe slabs is shown. In contrast to the small oscillation of the charge in the MnSe slab and in the inner QLs of $\mathrm{Bi}_{2} \mathrm{Se}_{3}$ a large charge redistribution is found in the QL adjacent to the interface pane. Such a change in the electron charge density within the first QL results in a substantial modification of the electrostatic potential which shows modulated band-bending behavior within the interfacial QL [Fig. 1(c)]. Owing to the strong modification of the first QL potential the localized states of the interfacial QL split off from the conduction band and spread across the gap (Fig. 2). These metallic states, if they are not suppressed-for example by means of disorder at the interface or chemical doping - can hinder the topological state manipulation. The interesting feature is that the topological Dirac state, being localized in the outermost QL of the free $\mathrm{Bi}_{2} \mathrm{Se}_{3}$ surface, survives upon the formation of the interface relocating to the second QL.

Additionally, two types of states arise at -0.6 to $-0.8 \mathrm{eV}$ in the local bulk energy gap: The gapped $(56 \mathrm{meV})$ interfacial
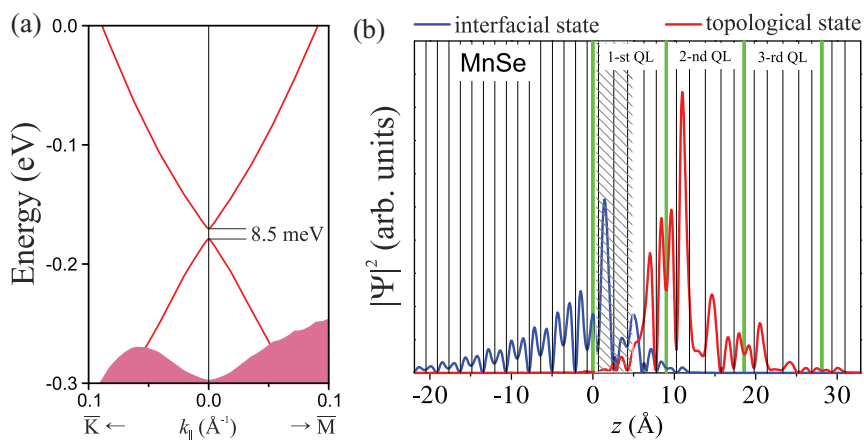

FIG. 3. (Color online) (a) A magnified view of the electronic structure of $\mathrm{Bi}_{2} \mathrm{Se}_{3} / \mathrm{MnSe}(111)$ corresponds to the green frame marked in Fig. 2. (b) Spatial localization of the topological (red) and interfacial (blue) states at the $\bar{\Gamma}$ point; the hatched area covers the $\mathrm{Bi}_{2} \mathrm{Se}_{3}$ layers with induced magnetization.

state and the degenerate at the $\bar{\Gamma}$-point two spin-split states, localized in the second QL, which are split off from the gap edges. The latter states are similar to those which reside near the bottom of the local valence band gap in the TIs of the $\mathrm{Bi}_{2} \mathrm{Se}_{3}$ family [they were studied in detail in $\mathrm{Sb}_{2} \mathrm{Te}_{3}$ (Ref. 31)] but here they appear at higher energy owing to the lower part of this gap being filled by the MnSe bulk states. The former, interfacial state, is similar to the state with a gap of $\sim 54 \mathrm{meV}$ found in Ref. 19, which was assumed as a gapped Dirac cone. The unoccupied $\bar{\Gamma}$ state lying in the local gap of the conduction band at $\approx 0.5 \mathrm{eV}$ (Fig. 2) also arises owing to the strong modification of the first QL potential which leads to the overall shift of the first QL states to lower energies. On the clean $\mathrm{Bi}_{2} \mathrm{Se}_{3}$ surface this state was recently discovered in angle-resolved monochromatic 2PPE measurement ${ }^{32}$ and DFT calculations ${ }^{32,33}$ at higher energy.

A magnified view of the Dirac state is shown in Fig. 3(a). As one can see, the cone is gapped at the $\bar{\Gamma}$ point. Figure $3(\mathrm{~b})$ shows that the topological state tends to leave the layers with induced magnetization. Thus the gap of $8.5 \mathrm{meV}$ in the Dirac cone is provided by an overlap of the topological and spin-polarized interfacial states within the first QL [Fig. 3(b)]. The induced magnetization at the interface is limited to three atomic layers of $\mathrm{Bi}_{2} \mathrm{Se}_{3}$. The magnetic moment on the $\mathrm{Bi}$ atoms (second atomic layer) is $0.04 \mu_{\mathrm{B}}$ while it is smaller on Se atoms, $\leqslant 0.01 \mu_{\mathrm{B}}$. Since the area of induced magnetization in the TI part of the system is mostly restricted to the three atomic layers closest to the interface plane [Fig. 3(b)], and the magnetic moments in these layers are small, the unoccupied state is only slightly influenced and nearly zero gapped. The latter fact also explains why the trivial states in the local valence band gap are not gapped - they leave a narrow area of induced magnetization being relocated into the second QL.

To elucidate the obtained results we develop an analytical model for the magnetic proximity effect in the TI/MI heterostructure, which is based on a recently proposed method to describe the formation of the bound in-gap electron states at the interface between a 3D TI and a normal insulator. ${ }^{34}$ Our model differs from an approach routinely used in the study of the TI/MI interface ${ }^{18}$ when the existence of the exchange term in the phenomenological Hamiltonian of the two-dimensional Dirac-like states is simply postulated. 
We write the full electron energy of the TI/MI contact in the following form:

$$
\begin{gathered}
\Omega=\int_{z>0} d \mathbf{r} \Theta^{+}(\mathbf{r}) H_{t}(-i \nabla) \Theta(\mathbf{r}) \\
+\int_{z<0} d \mathbf{r} \Phi^{+}(\mathbf{r}) H_{m}(-i \nabla) \Phi(\mathbf{r})+\Omega_{I}, \\
\Omega_{I}=\int d \mathbf{r}\left[\Theta^{+}(\mathbf{r}) V(\mathbf{r}) \Phi(\mathbf{r})+\Phi^{+}(\mathbf{r}) V^{+}(\mathbf{r}) \Theta(\mathbf{r})\right] .
\end{gathered}
$$

In the TI half-space $(z>0)$, the four band $\mathbf{k} \cdot \mathbf{p}$ Hamiltonian $H_{t}$, proposed in Ref. 35 for the narrow-gap semiconductors of the $\mathrm{Bi}_{2} \mathrm{Se}_{3}$ family, describes the low energy and long wavelength bulk electron states near the $\Gamma$ point of the Brillouin zone. In the MI half-space $(z<0)$, the electron states are modeled within the effective mass approximation by the four band Hamiltonian without SOI, $H_{m}$. The intrinsic magnetization of the MI is assumed to be perpendicular to the TI/MI interface plane $(z=0)$. The spinors $\Theta(\mathbf{r})$ and $\Phi(\mathbf{r})$ are smooth and continuous envelope functions in the right and left half-spaces, respectively. The term $\Omega_{I}$ involves intermixing of the TI and MI electron states at the interface via the effective potential of hybridization $V(\mathbf{r})$.

Following the variational procedure, ${ }^{34}$ one can show that the energy functional [Eqs. (1) and (2)] has two distinct bound states: ordinary and topological states with the exponentially decaying functions far from the interface: $\Phi(z \rightarrow-\infty)=0$ and $\Theta(z \rightarrow \infty)=0$. One of them, the interfacial ordinary state, is localized near the interface and exponentially decays into the TI half-space with a scale length $z_{o}$. The charge redistribution near the interface causes a significant shift of the ordinary state spectrum relative to the TI bulk energy spectrum. ${ }^{34}$ As seen from the Boltzmann fit for $\Delta V_{\mathrm{TI}}$ in Fig. 1(c), in the $\mathrm{Bi}_{2} \mathrm{Se}_{3} / \mathrm{MnSe}$ system, the band bending on the TI side of the interface is $\sim 0.8 \mathrm{eV}$ so that the interfacial ordinary state is sunk into the region of the bulk valence band. Furthermore, due to the hybridization (2) with the orbitals of the MI outermost layer that exhibits the out-of-plane magnetization $M$, the interfacial ordinary state becomes spin polarized.

Another state at the interface, the topological state, is not directly influenced by the interface potential since $\Theta_{t}(z=$ $0)=0$. This topological state is remote from the interface at the distance $z_{t}<z_{o}$; note that the lengths $z_{o}$ and $z_{t}$ are determined by the material parameters of the TI. Thus the topological state experiences a magnetic effect of MI through the magnetic proximity effect, when the exchange field induced by the spin polarization of the interfacial ordinary state penetrates deep into the TI half-space. The magnetic proximity-induced gap at the Dirac point in the electron spectrum of the topological state is estimated as $\Delta \sim J M S|V|^{2}$, where $J$ is the exchange interaction strength in the TI; $S \sim \int_{0}^{\infty} d z\left|\Theta_{o} \Theta_{t}\right|^{2}$ is the overlap integral of the ordinary and topological states in the TI. The analytical results will be presented in detail elsewhere.

The calculated orientation of the electron spin for the topological state is presented in Fig. 4. One can see, at large momentum the gapped state demonstrates in-plane spin polarization (the out-of-plane component $S_{z}$ is negligibly small) with a positive (clockwise) spin helicity in the upper (a)

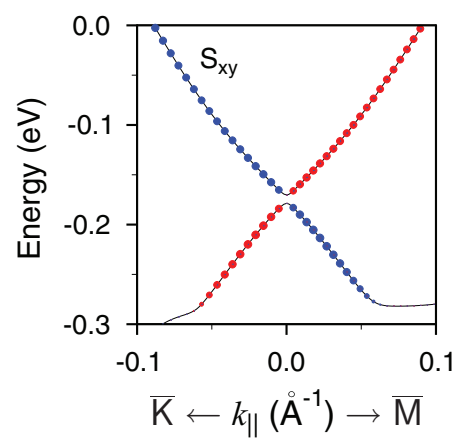

(b)

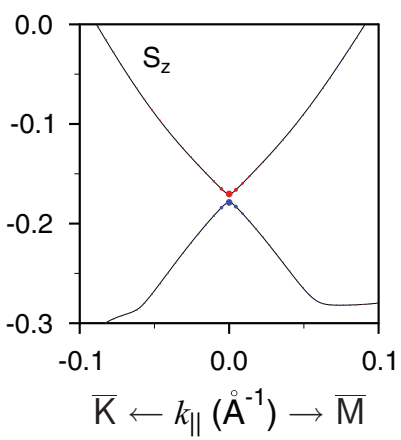

FIG. 4. (Color online) Spin polarization in the topological state given as Cartesian spin projections $S_{x y}$ (a) and $S_{z}$ (b).

band and a negative helicity in the lower band. In the close vicinity of the $\bar{\Gamma}$ point the in-plane spin polarization vanishes while the out-of-plane spin component arises so that the upper and lower bands separated by the magnetic gap demonstrate opposite sign of the spin polarization. Thus, the spin texture of the gapped Dirac state at the $\mathrm{Bi}_{2} \mathrm{Se}_{3} / \mathrm{MnSe}(111)$ interface is similar to that found for the magnetic atom doped TI. ${ }^{36}$

\section{CONCLUSION}

In summary, on the basis of DFT calculations performed on the $\mathrm{Bi}_{2} \mathrm{Se}_{3} / \mathrm{MnSe}$ system, we scrutinized the magnetic proximity effect at the 3D TI/MI interface. We have shown that the charge redistribution and the mixing of the TI orbitals with the MI orbitals at the interface cause drastic modifications of the electronic structure near the TI/MI interface. The calculation data reveal the presence of the interfacial ordinary state confined within the adjacent interfacial QL of TI which slowly decays into a MI. This state is shifted downwards to the local energy gap owing to the near-interface band bending. The state is gapped and spin polarized due to the hybridization with the MI states. On the other hand, the topological state is mainly localized in the second QL showing an overlap with the interfacial ordinary state which in this way mediates indirect exchange coupling between the MI and the topological state. The topological state acquires the energy gap at the Dirac point proportional to the overlap of the topological and interfacial ordinary states. The key DFT results are supported by the analytical model. The unveiled mechanism of the magnetic proximity effect in the TI/MI structure provides a pathway to integrate TIs in spintronic devices.

\section{ACKNOWLEDGMENTS}

We acknowledge partial support from the Basque Country Government, Departamento de Educación, Universidades e Investigación (Grant No. IT-366-07), the Spanish Ministerio de Ciencia e Innovación (Grant No. FIS2010-19609-C02-00), and the Ministry of Education and Science of Russian Federation (Grant No. 2.8575.2013). 
${ }^{1}$ M. Z. Hasan and C. L. Kane, Rev. Mod. Phys. 82, 3045 (2010).

${ }^{2}$ X.-L. Qi, R. Li, J. Zang, and S.-C. Zhang, Science 323, 1184 (2009).

${ }^{3}$ T. Fujita, M. B. A. Jalil, and S. G. Tan, Appl. Phys. Express 4, 094201 (2011).

${ }^{4}$ L. Oroszlány and A. Cortijo, Phys. Rev. B 86, 195427 (2012).

${ }^{5}$ P. Wei, F. Katmis, B. A. Assaf, H. Steinberg, P. Jarillo-Herrero, D. Heiman, and J. S. Moodera, Phys. Rev. Lett. 110, 186807 (2013). ${ }^{6}$ C.-Z. Chang, J. Zhang, X. Feng, J. Shen, Z. Zhang, M. Guo, K. Li, Y. Ou, P. Wei, L.-L. Wang, Z.-Q. Ji, Y. Feng, S. Ji, X. Chen, J. Jia, X. Dai, Z. Fang, S.-C. Zhang, K. He, Y. Wang, L. Lu, X.-C. Ma, and Q.-K. Xue, Science 340, 167 (2013).

${ }^{7}$ L. A. Wray, S.-Y. Xu, Y. Xia, D. Hsieh, A. V. Fedorov, Y. S. Hor, R. J. Cava, A. Bansil, H. Lin, and M. Z. Hasan, Nat. Phys. 7, 32 (2011).

${ }^{8}$ M. R. Scholz, J. Sánchez-Barriga, D. Marchenko, A. Varykhalov, A. Volykhov, L. V. Yashina, and O. Rader, Phys. Rev. Lett. 108, 256810 (2012).

${ }^{9}$ J. Honolka, A. A. Khajetoorians, V. Sessi, T. O. Wehling, S. Stepanow, J.-L. Mi, B. B. Iversen, T. Schlenk, J. Wiebe, N. B. Brookes, A. I. Lichtenstein, Ph. Hofmann, K. Kern, and R. Wiesendanger, Phys. Rev. Lett. 108, 256811 (2012).

${ }^{10}$ M. Ye, S. V. Eremeev, K. Kuroda, E. E. Krasovskii, E. V. Chulkov, Y. Takeda, Y. Saitoh, K. Okamoto, S. Y. Zhu, K. Miyamoto, M. Arita, M. Nakatake, T. Okuda, Y. Ueda, K. Shimada, H. Namatame, M. Taniguchi, and A. Kimura, Phys. Rev. B 85, 205317 (2012).

${ }^{11}$ Q. Liu, C.-X. Liu, C. Xu, X.-L. Qi, and S.-C. Zhang, Phys. Rev. Lett. 102, 156603 (2009).

${ }^{12}$ J. Henk, M. Flieger, I. V. Maznichenko, I. Mertig, A. Ernst, S. V. Eremeev, and E. V. Chulkov, Phys. Rev. Lett. 109, 076801 (2012).

${ }^{13}$ Y. Tanaka, T. Yokoyama, and N. Nagaosa, Phys. Rev. Lett. 103, 107002 (2009).

${ }^{14}$ G. Rosenberg and M. Franz, Phys. Rev. B 85, 195119 (2012).

${ }^{15}$ V. N. Men'shov, V. V. Tugushev, and E. V. Chulkov, JETP Lett. 94, 629 (2011).

${ }^{16}$ Z. L. Li, J. H. Yang, G. H. Chen, M.-H. Whangbo, H. J. Xiang, and X. G. Gong, Phys. Rev. B 85, 054426 (2012).

${ }^{17}$ I. Vobornik, U. Manju, J. Fujii, F. Borgatti, P. Torelli, D. Krizmancic, Y. S. Hor, R. J. Cava, and G. Panaccione, Nano Lett. 11, 4079 (2011).
${ }^{18}$ I. Garate and M. Franz, Phys. Rev. Lett. 104, 146802 (2010).

${ }^{19}$ W. Luo and X.-L. Qi, Phys. Rev. B 87, 085431 (2013).

${ }^{20} \mathrm{G}$. Kresse and J. Hafner, Phys. Rev. B 48, 13115 (1993).

${ }^{21}$ G. Kresse and J. Furthmüller, Comput. Mater. Sci. 6, 15 (1996).

${ }^{22}$ J. P. Perdew, K. Burke, and M. Ernzerhof, Phys. Rev. Lett. 77, 3865 (1996).

${ }^{23}$ P. E. Blöchl, Phys. Rev. B 50, 17953 (1994).

${ }^{24}$ G. Kresse and D. Joubert, Phys. Rev. B 59, 1758 (1999).

${ }^{25}$ D. D. Koelling and B. N. Harmon, J. Phys. C 10, 3107 (1977).

${ }^{26}$ A. I. Liechtenstein, V. I. Anisimov, and J. Zaanen, Phys. Rev. B 52, R5467 (1995).

${ }^{27}$ P. Klosowski, T. M. Giebultowicz, J. J. Rhyne, N. Samarth, H. Luo, and J. Furdyna, J. Appl. Phys. 69, 6109 (1991).

${ }^{28}$ S. J. Youn, J. Magn. 10, 71 (2005).

${ }^{29}$ P. Amiri, S. J. Hashemifar, and H. Akbarzadeh, Phys. Rev. B 83, 165424 (2011)

${ }^{30}$ W. Tang, E. Sanville, and G. Henkelman, J. Phys.: Condens. Matter 21, 084204 (2009).

${ }^{31}$ C. Pauly, G. Bihlmayer, M. Liebmann, M. Grob, A. Georgi, D. Subramaniam, M. R. Scholz, J. Sánchez-Barriga, A. Varykhalov, S. Blügel, O. Rader, and M. Morgenstern, Phys. Rev. B 86, 235106 (2012).

${ }^{32}$ D. Niesner, Th. Fauster, S. V. Eremeev, T. V. Menshchikova, Yu. M. Koroteev, A. P. Protogenov, E. V. Chulkov, O. E. Tereshchenko, K. A. Kokh, O. Alekperov, A. Nadjafov, and N. Mamedov, Phys. Rev. B 86, 205403 (2012).

${ }^{33}$ S. V. Eremeev, I. V. Silkin, T. V. Menshchikova, A. P. Protogenov, and E. V. Chulkov, JETP Lett. 96, 780 (2013).

${ }^{34}$ V. N. Men'shov, V. V. Tugushev, and E. V. Chulkov, Pis'ma Zh. Eksp. Teor. Fiz. 97, 297 (2013) [JETP Lett. 97, 258 (2013)].

${ }^{35}$ H. Zhang, C.-X. Liu, X.-L. Qi, X. Dai, Z. Fang, and S.-C. Zhang, Nat. Phys. 5, 438 (2009).

${ }^{36}$ S.-Y. Xu, M. Neupane, Ch. Liu, D. Zhang, A. Richardella, L. A. Wray, N. Alidoust, M. Leandersson, Th. Balasubramanian, J. Sánchez-Barriga, O. Rader, G. Landolt, B. Slomski, J. H. Dil, J. Osterwalder, T.-R. Chang, H.-T. Jeng, H. Lin, A. Bansil, N. Samarth, and M. Z. Hasan, Nat. Phys. 8, 616 (2012). 